\title{
LA DIFICIL TAREA DE SELECCIONAR, ORGANIZAR Y SECUENCIAR LOS CONTENIDOS AL MOMENTO DE PLANIFICAR. Los Temas Escolares de la Geografía
}

\section{THE CHALLENGE OF SELECTING, ORGANIZING AND SEQUENCING CONTENTS AT THE TIME OF PLANNING. Geography topics at school.}

\author{
Prof./ Lic. Lucía Iris MERETZ \\ Prof, Adjunta de Didáctica Específica y Pasantía \\ Profesorado en Geografía - Departamento de \\ Geografía \\ Facultad de Humanidades - UNNE \\ irismeretz@hotmail.com
}

\section{RESUMEN}

En el contexto actual de acelerados cambios sociales, económicos, políticos, culturales y ambientales, la enseñanza de la geografía no puede desentenderse de los múltiples aportes provenientes de los distintos ámbitos de la producción científica y cultural.

Junto con la necesidad de incorporar perspectivas, temáticas y metodologías acordes con los nuevos desafíos de la enseñanza de la geografía, se plantea la pregunta de cuáles son los recortes conceptuales más significativos y de qué modo organizarlos y presentarlos en clase para lograr que los jóvenes construyan el conocimiento escolar y tengan una mirada crítica sobre el complejo acontecer social. La construcción del conocimiento escolar requiere, en cada contexto particular de enseñanza, de una serie de mediaciones pedagógicas que transformen los saberes provenientes del campo disciplinar y cultural en contenidos susceptibles de ser enseñados, aprendidos y evaluados.

\begin{abstract}
:
In a fast-changing world in terms of society, economy, politics, culture and environment, the teaching of geography cannot disregard contributions from the scientific and cultural community.

Along with the necessity of introducing views, issues and methodologies in accordance with the new challenges that the teaching of geography implies, there appears the question of which the most significant selection of conceptual contents is, and the way such contents should be organized and presented in a class so that the youth may construct academic knowledge and have a critical look at the complex social reality. The construction of academic knowledge involves, in each particular teaching context, a series of pedagogical mediations which may transform disciplinary and cultural knowledge into concepts susceptible to being taught, learnt and evaluated.
\end{abstract}

PALABRAS CLAVE:

Concepciones de enseñanza; Aprendizaje Significativo; Selección de Contenidos; Secuencias de contenidos; Relación Sociedad-Naturaleza.

\section{KEY WORDS:}

Teaching conceptions; significant learning; selection of contents; sequence of contents; nature-society relation. 
Revista Geográfica Digital. IGUNNE. Facultad de Humanidades. UNNE. Año 10. № 20.

Julio - Diciembre 2013. ISSN 1668-5180 Resistencia, Chaco

\section{Los Temas Escolares De La Geografía}

"A lo largo de la historia de la Geografía se han considerado diversos ejes temáticos como los hilos conductores de la disciplina. Así; durante décadas la relación hombre-medio estuvo en el centro de la escena, en sus distintas variantes.' con una fuerte influencia de los componentes físicosbiológicos por sobre el hombre, o con un mayor margen de acción por parte del hombre frente a las posibilidades que la naturaleza le ofrece.

La primera variante, donde se analizan sistemáticamente los componentes del medio físico por separado, continúa ocupando un papel importante en la Geografía escolar; la segunda versión, donde la historia cobra mayor importancia, también se manifiesta actualmente en la enseñanza de la geografía a través de los estudios regionales. Las dos han demostrado su fracaso en la escuela por diversas razones, entre las que se destacan la fragmentación propia de la primera línea y la falta de explicación, que se traduce en una descripción interminable, sin sentido y también desintegrada, asociada a la segunda línea."(1)

Todos los que hemos transitado gran parte del siglo XX por las aulas, tanto en calidad de alumnos como de docentes, sabemos que la precedente afirmación es completamente verdadera.

Las clases de Geografía consideraron como ejes centrales de su enseñanza, fundamentalmente, el estudio de los componentes físico-biológicos y su acción sobre el hombre y, más tarde, las regiones geográficas.

Uno y otro eje pocas veces permitieron la integración de los elementos que en ellos se mencionaban y ubicaban. Tampoco facilitaron la posibilidad de comprender el por qué de la organización espacial estudiada, la relación con los intereses y necesidades políticos, económicos, ideológicos o culturales de la sociedad que la ocupaba y dicha configuración espacial.

Como dicen muchos autores, los interminables inventarios de elementos debían ser localizados en un mapa y recordados para su evaluación.

Es necesario revisar estas cuestiones. Es necesario dotarlas de nueva significación, de un sentido nuevo que permita al alumno descubrir la íntima relación existente entre el espacio construido y la sociedad que lo ocupa, entre los elementos naturales o estructurales de un lugar determinado y los aspectos dinámicos existentes, aquellos que son fruto de la obra de la sociedad.

Dice Reboratti: "La geografía que se desarrolla actualmente en los ámbitos académicos de todo el mundo maneja, desde su particular punto de vista, tres grandes líneas temáticas: el ambiente, la sociedad y la organización del territorio. Estos no son obviamente grupos aislados, sino que están íntimamente relacionados, y su separación responde sólo a una estrategia analítica. El acercamiento al ambiente y la sociedad son especificaciones necesarias, pasos previos para comprender la organización del territorio, pero esta desagregación es, en cuanto se la analiza, ilusoria."(2)

\section{El ambiente}

"La noción medioambiental y sus consecuencias deben insertarse en los contenidos fundamentales tanto inter como intradisciplinarios en todos los ciclos de la enseñanza.

Debe ser abordado como un proceso que consiste en ver qué valores y conocimientos aporta el alumno con el fin de incrementarlos. El proceso implica dinamismo y muestra la necesidad de una continua actualización.

El papel más importante es demostrar que la conservación es en beneficio del medio ambiente, por
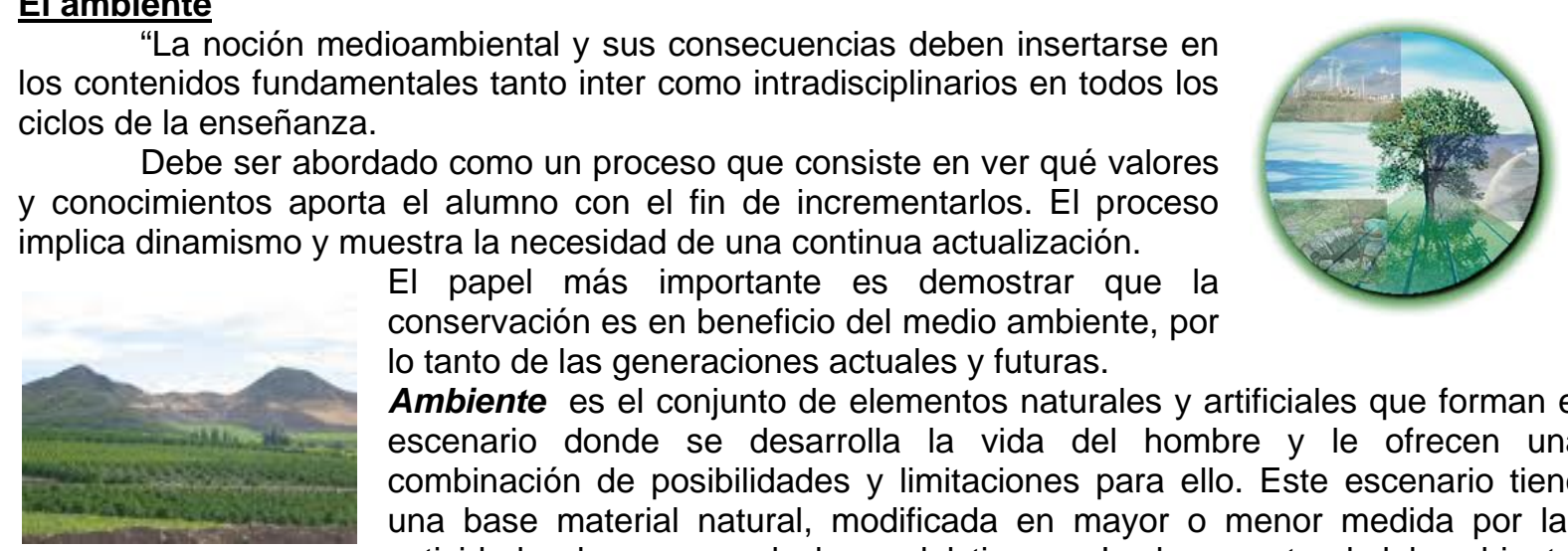
lo tanto de las generaciones actuales y futuras.

Ambiente es el conjunto de elementos naturales y artificiales que forman el escenario donde se desarrolla la vida del hombre y le ofrecen una combinación de posibilidades y limitaciones para ello. Este escenario tiene una base material natural, modificada en mayor o menor medida por las actividades humanas a lo largo del tiempo. La base natural del ambiente, debería ser analizada no como un inventario de datos físicos agrupados en unidades estrictamente separadas (relieve, clima, etc.), sino como un sistema dinámico -el ecosistema humano- que no es inmutable, sino que justamente se caracteriza por su movilidad. (Reboratti, C.)

Publicado en formato digital: Prof./ Lic. Lucía Iris Meretz. LA DIFICIL TAREA DE SELECCIONAR, ORGANIZAR Y SECUENCIAR LOS CONTENIDOS AL MOMENTO DE PLANIFICAR. Los Temas Escolares de la Geografía. Revista Geográfica Digital. IGUNNE. Facultad de Humanidades. UNNE. Año 10. № 20. Julio Diciembre 2013. ISSN 1668-5180 Resistencia, Chaco. En: http://hum.unne.edu.ar/revistas/geoweb/default.htm 
Como se observa, se trata de un conjunto de elementos naturales y artificiales integrados, articulados históricamente. Hablamos de una mutua relación: las acciones de la sociedad impactan y actúan sobre lo natural; lo natural ofrece limitaciones o problemas que la sociedad padece, procura controlar o intenta protegerse de ellas.

Todos vivimos en un entorno específico, formado por la acción de la sociedad sobre un medio natural original. Ese ambiente es tanto o más artificial en la medida en que el hombre actúa sobre él.

"El grado de artificialización es alto en las grandes ciudades, mediano en los pueblos, bajo en las zonas rurales, pero siempre presente. A la inversa, cualquiera sea ese nivel siempre, por debajo del ambiente directamente perceptible, existe una base relacionada con los factores de la naturaleza, un soporte natural. " (Reboratti, C.)
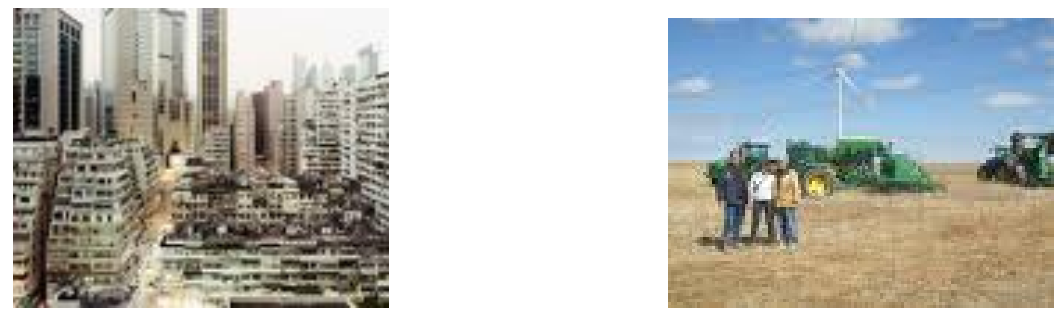

\section{La sociedad}

Aquí diferenciamos el concepto de sociedad del de población. El término población hace referencia a cuestiones cuantitativas y estadísticas: datos de población, pirámides poblacionales, crecimiento vegetativo, tasas de mortalidad y natalidad son algunos de los aspectos que el concepto contiene.

"Pero la sociedad admite otras miradas: físicamente es esa población, pero a esto se le suman las relaciones y organizaciones que los individuos y grupos sociales establecen entre sí en los diferentes contextos territoriales y temporales."(Reboratti, C.)

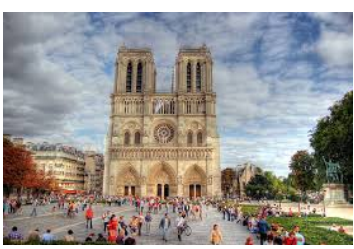

Incluimos los conceptos de grupos, clases, asociaciones, estructura, composición, conflictos (políticos, económicos y sociales que la sociedad vive). Cuando hablamos de conflictos sociales nos referimos a los enfrentamientos de los diferentes grupos o asociaciones en relación con cuestiones vinculadas al poder al uso o distribución de los recursos, a la oposición de ideas religiosas, etc.

Dentro del análisis de los factores económicos, culturales y sociales hay que evitar las aproximaciones dogmáticas y rígidas y entender por ejemplo, a los sistemas económicos como elementos dinámicos.

Desde ese punto de vista es muy importante hacer conocer al alumno las nuevas tendencias de la economía tanto nacional como global: la internacionalización, la aparición de grandes compañías multinacionales, la creciente importancia del sector terciario de la economía informal, las nuevas de los formas de producción agrícola e industrial, la relación con el uso de los recursos naturales y el medio ambiente.

\section{La organización del territorio (relación sociedad-naturaleza)}

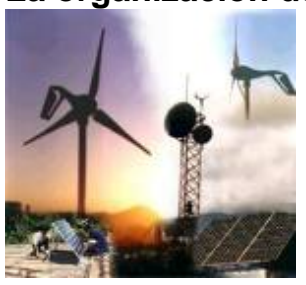

En esta línea temática "se abordará el estudio de los grupos sociales y sus formas de organización con los modos en que se distribuyen y ocupan el espacio, se desplazan a través del mismo y lo organizan modificándolo. Las modificaciones del espacio natural responden a diferentes tipos de actividades: las económicas, las políticas y las sociales, que, estrechamente vinculadas entre sí configuran distintos modos de organización social" (3)

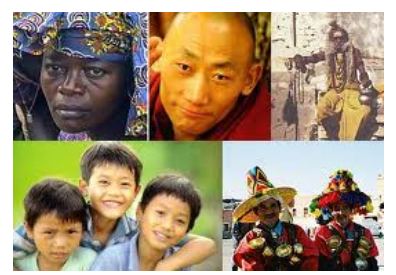

El objetivo es mostrar y explicar cómo la sociedad, en diversas escalas y con diversos medios, construye un espacio propio, y va modificando ese ambiente natural al que nos referíamos antes. Como elemento de mediación entre la sociedad y su territorio, adquiere relevancia el tema de

Publicado en formato digital: Prof./ Lic. Lucía Iris Meretz. LA DIFICIL TAREA DE SELECCIONAR, ORGANIZAR Y SECUENCIAR LOS CONTENIDOS AL MOMENTO DE PLANIFICAR. Los Temas Escolares de la Geografía. Revista Geográfica Digital. IGUNNE. Facultad de Humanidades. UNNE. Año 10. № 20. Julio Diciembre 2013. ISSN 1668-5180 Resistencia, Chaco. En: http://hum.unne.edu.ar/revistas/geoweb/default.htm 
la tecnología, aquel grupo de instrumentos y habilidades que le permiten a la sociedad aumentar su capacidad productiva y transformadora.

En este contexto deben ser comprendidos los temas propuestos en torno al eje sociedadnaturaleza. Algunos ejemplos de interés son:

- La valorización, apropiación y manejo de los recursos naturales.

- El desarrollo sustentable.

- Las condiciones y efectos del desarrollo de los sistemas productivos agropecuarios y forestales.

- La dimensión ambiental de los procesos productivos.

- Los problemas ambientales en sus distintas escalas (local, regional, nacional, global).

- La dinámica demográfica y la satisfacción de las necesidades.

- Los cambios climáticos y las transformaciones productivas.

- Los desastres naturales y su impacto social."
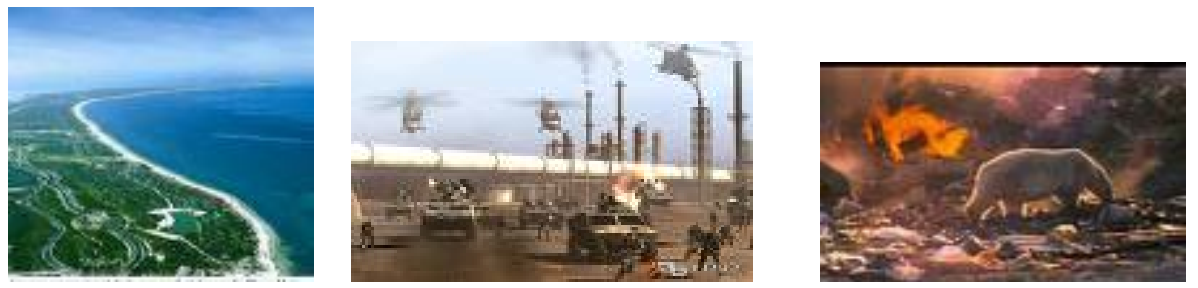

Esta relación es fundamental para la Geografía, así como para otras Ciencias Sociales. Desde sus comienzos, los hombres han obtenido de la naturaleza una serie de elementos que les permitieron satisfacer sus necesidades básicas. Esta relación fue modificándose a lo largo del tiempo; así, en la historia de la Geografía, hubo momentos en los que la naturaleza era la que imponía sus condiciones al hombre, mientras en otros, era el hombre el que tenía diferentes posibilidades de transformarla.

Para analizar la relación sociedad-naturaleza seguiremos el planteo del ecólogo argentino G. Gallopín, quien reconoce dos tipos principales de factores:

"El conjunto de acciones humanas que inciden sobre el sistema ecológico y el conjunto de respuestas ecológicas a esas acciones". (4)

"Las acciones humanas incluyen la tecnología, considerada en su sentido más amplio, definida como el conjunto de herramientas, materiales, conocimientos y habilidades empleados para satisfacer las necesidades de la sociedad y para establecer sus interacciones con el ambiente. La tecnología condiciona el 'qué hacer' y 'cómo hacer' de la sociedad (Herrera, 1980). Sin embargo, la inclusión de las acciones humanas y las respuestas ecológicas es, en general, insuficiente como marco de referencia para comprender y solucionar los problemas ecológicos. " (5)

"Desde el punto de vista del sistema ecológico, se hace importante conocer no solamente qué respuestas ecológicas se producen frente a las acciones, y cómo afectan al sistema humano (disminución de la producción, abandono de la tierra, deterioro de la salud, etc.), sino también cuáles son los elementos ecológicos afectados por las acciones humanas, cómo y por qué se producen las respuestas, lo que depende de las estructuras de los procesos ecológicos relevantes. (6)
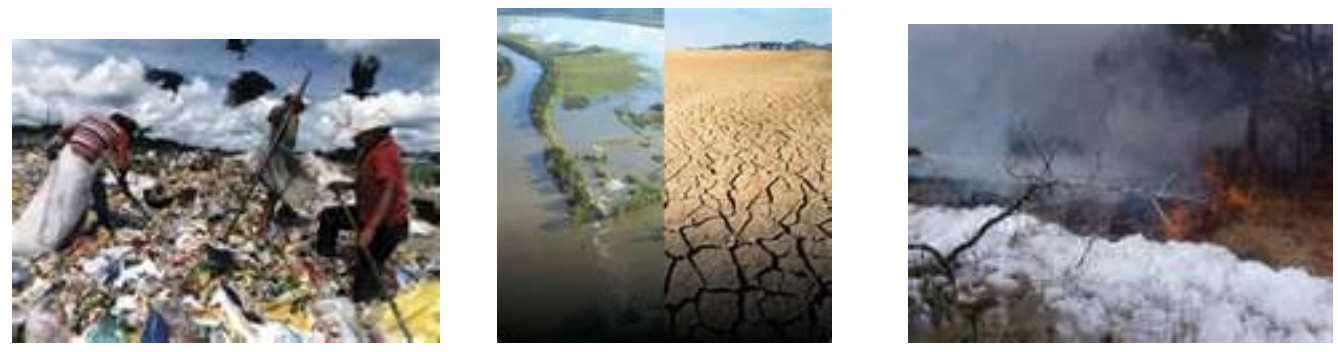

Publicado en formato digital: Prof./ Lic. Lucía Iris Meretz. LA DIFICIL TAREA DE SELECCIONAR, ORGANIZAR Y SECUENCIAR LOS CONTENIDOS AL MOMENTO DE PLANIFICAR. Los Temas Escolares de la Geografía. Revista Geográfica Digital. IGUNNE. Facultad de Humanidades. UNNE. Año 10. No 20. Julio Diciembre 2013. ISSN 1668-5180 Resistencia, Chaco. En: http://hum.unne.edu.ar/revistas/geoweb/default.htm 
A partir de este aporte se puede avanzar, haciendo referencia a tres cuestiones centrales en la enseñanza de la geografía:

- La organización social de los territorios.

- La organización política de los territorios.

- La organización económica de los territorios.

La cuestión del papel que desempeña la sociedad con respecto a la organización del territorio en el cual vive admite varias visiones, obviamente interrelacionadas. Una es la de la propia organización social del territorio (cómo y por qué los distintos individuos o grupos de individuos se distribuyen en él); otra es la organización económica del territorio (cómo y para qué los individuos organizan su actividad productiva en forma diferencial sobre el espacio que habitan) y otra finalmente es la organización política del territorio (cómo y por qué el individuo y los grupos sociales se adueñan del territorio). Aunque las tres dimensiones confluyen hacia lo que podríamos llamar en líneas generales 'la organización del espacio', conviene a los fines didácticos separar sus elementos, para hacerlos más comprensibles." (7)

\section{La organización social de los territorios}

La población se distribuye en el territorio de acuerdo con varias características, por ejemplo: afinidad cultural, historia común, necesidad económica, capacidad de acceso a la tierra o control de distancias hacia puntos importantes. Estos factores actúan tanto solos como en forma combinada, y son manejados a veces por los individuos, a veces por los grupos sociales más pequeños, a veces por los grupos de poder, y también son regulados por los sistemas de control social de distinta índole.

\section{La organización política de los territorios}

La organización política del territorio incluye, por ejemplo, las reglas de convivencia, regulación y control de la sociedad sobre el espacio que ocupa; su compartimentalización en diversos "territorios" que poseen un sistema de gobierno específico: gobiernos municipales, provinciales y nacionales. El trabajo con fronteras y límites entre países y grupos sociales, etc.

El tema territorial es especialmente complejo en el nivel político. La sociedad se adueña del espacio y lo controla por diversos métodos, y ese control lo ejerce a través de una cierta organización política, regulada por las normas de convivencia básicas de ese grupo social.

\section{La organización económica de los territorios}

La actividad económica (entendida como las acciones que se desarrollan en la sociedad alrededor de la producción, distribución y consumo de bienes y servicios) genera sobre el territorio una trama de instalaciones y relaciones que se puede analizar a diversas escalas y niveles de profundidad y complejidad, desde los aspectos más visibles y las relaciones más directas a las estructuras más complejas y globales.

Del mismo modo que en el eje sociedad-naturaleza, es posible distinguir en el entorno de la organización del espacio una serie de temas. Aquí se presentan algunos a modo de ejemplo:

- La cuestión general de la localización y sus casos particulares.

- Los procesos de urbanización y las contradicciones de la estructura urbana.

- Transporte y comunicaciones en la economía global.

- La dimensión territorial de las políticas estatales.

- Los circuitos de producción y acumulación.

- Las ciudades como espacios de consumo y entretenimiento.

- Los intercambios comerciales en la economía global.

- La actividad industrial como organizadora de los territorios.

- Influencia del avance tecnológico.

\section{Un método crítico, abierto y creador a la hora de planificar}

En el mundo actual, acelerado y repleto de imágenes y datos es necesario reencontrar el hombre integral que incluye: intelecto, emociones, afectos, espíritu.

La pregunta sobre qué enseñar sólo puede surgir luego de haber respondido con responsabilidad a la pregunta sobre qué mundo debe surgir de esta crisis en la que se está inmerso.

Publicado en formato digital: Prof./ Lic. Lucía Iris Meretz. LA DIFICIL TAREA DE SELECCIONAR, ORGANIZAR Y SECUENCIAR LOS CONTENIDOS AL MOMENTO DE PLANIFICAR. Los Temas Escolares de la Geografía. Revista Geográfica Digital. IGUNNE. Facultad de Humanidades. UNNE. Año 10. No 20. Julio Diciembre 2013. ISSN 1668-5180 Resistencia, Chaco. En: http://hum.unne.edu.ar/revistas/geoweb/default.htm 
La educación debe tender a la adquisición de conocimientos, la estructuración de la inteligencia, el desarrollo de la capacidad crítica y el autoconocimiento, el dominio de tendencias destructivas, el fomento de la creatividad y la imaginación, el desempeño de un papel responsable en la sociedad. Además debe ayudar a las personas a adaptarse e prepararse para el cambio, y permitirles recuperar la concepción global del mundo y la humanidad.

El docente abierto ayuda a formular preguntas, a explorar en el mundo interior, a distinguir modelos y relaciones, a "admitir nuevas alternativas por muy singulares que parezcan; es un facilitador permanente, pero sabe que no es la razón principal. El auténtico educador está predispuesto a admitir sus propias equivocaciones, a reconocer en su alumno una realidad diferente a la propia porque la 'autoridad' está en su propia voz interior".

La idea que se tenga acerca de cuál es el campo de estudio de la geografía y su estructura lógica, así como la relevancia de determinados conceptos para alcanzar aprendizajes significativos, la concepción de aprendizaje y las finalidades que se otorguen a estos saberes, influyen decisivamente en la enseñanza de la geografía. al respecto:

En relación con el objeto de estudio de la geografía, son muchas las definiciones formuladas

- La distribución de los fenómenos físicos y humanos sobre la superficie terrestre, las causas de esa repartición y las relaciones que entre ellos se producen.

- La organización espacial como un sistema en el que el hombre es un elemento más de él.

- El espacio geográfico como una instancia de lo social, en la que los hombres participan, como sujetos de la sociedad, en la producción y/o reproducción de dicho espacio.

- Quién consigue un determinado producto, dónde, cómo...

Estas concepciones, que estructuran la enseñanza de la disciplina, remiten a los fundamentos epistemológicos que, de manera implícita o explícita, están presentes en la práctica docente. Por ello es necesario tener en cuenta los elementos teóricos que se desprenden de los diferentes aportes epistemológicos, con el objetivo de que guíen la conceptualización y permitan una redefinición de la geografía que se enseña.

Para resignificar la enseñanza de la geografía es necesario además de tener en cuenta el qué y el dónde, preguntarnos por el "cómo", el "para que", el "para quién" y el "por qué".

Los métodos activos tienen que dar como resultado, según Patrik Bailey, una aceleración y profundización del aprendizaje y no un retraso del mismo. (8)

En la aplicación de métodos activos es necesario manifestarse a favor de una enseñanza selectiva de los contenidos geográficos, reemplazando el aprendizaje de tipo memorístico por el razonamiento, la resolución de situaciones problemáticas e incluso el método del juego, mediante la estrategia pedagógica del aprender descubriendo.

Para el trabajo en el aula es necesario contar con recursos didácticos que denominamos experiencias de aprendizaje. Las experiencias de aprendizaje se planifican simultáneamente con una selección de contenidos, debido a la estrecha relación entre ambos aspectos.

"Una geografía renovada debería tener como objetivo fundamental brindar herramientas para la comprensión de la realidad inmediata y mediata del alumno" (9)

\section{La importancia de la planificación}

Planificar el proceso de enseñanza aprendizaje significa tomar decisiones. Estas decisiones pueden tener el carácter de provisorias o definitivas, las cuales afectan directamente el logro de los objetivos educativos que la escuela se plantea alcanzar con los alumnos y alumnas

"Podemos definir a la planificación como el proceso mental que orienta la acción en una dirección determinada y que contempla los medios necesarios para alcanzar el fin. Este proceso mental puede ser representado a través de un diseño capaz de informarnos a nosotros mismos y también a otros sobre los alcances del plan o proyecto trazado". (Ruth Harf)

Al planificar el trabajo en aula, por ejemplo, el docente decide realizar ciertas acciones de una determinada forma, con unos recursos didácticos y no con otros, en un lapso de tiempo que estima conveniente para realizar esa acción, y lo más importante, con una finalidad bien precisa.

Toda planificación de aula refleja, sin lugar a dudas el posicionamiento que tiene el docente frente a la tarea de enseñar la disciplina y ante la manera de concebir al alumno y a su aprendizaje.

Publicado en formato digital: Prof./ Lic. Lucía Iris Meretz. LA DIFICIL TAREA DE SELECCIONAR, ORGANIZAR Y SECUENCIAR LOS CONTENIDOS AL MOMENTO DE PLANIFICAR. Los Temas Escolares de la Geografía. Revista Geográfica Digital. IGUNNE. Facultad de Humanidades. UNNE. Año 10. No 20. Julio Diciembre 2013. ISSN 1668-5180 Resistencia, Chaco. En: http://hum.unne.edu.ar/revistas/geoweb/default.htm 
Las alternativas de educación que considere el profesor, las estrategias que utilice, las prácticas de aprendizaje que priorice, los recursos didácticos que aproveche, las propuestas de cambios en la evaluación de esos aprendizajes, etc., constituyen factores de gran influencia en la enseñanza de los espacios geográficos indistintas escalas.

Al enfrentar la planificación como una tarea ineludible surgen diversas interrogantes por ejemplo. ¿Para qué planificar? ¿Cómo hacerlo? ¿Quiénes planifican? ¿En qué momento? ¿Qué elementos son necesarios considerar al planificar? ¿Existe un Modelo de planificación?

La planificación que realice un profesor determinado para su curso, deberá tener como marco de referencia el Proyecto Educativo Institucional (PEI) o Proyecto Educativo Comunitario (PEC) de la escuela. Esta herramienta de trabajo es el sello particular de la escuela. También es indispensable considerar el calendario escolar, las jornadas de perfeccionamiento, reuniones de trabajo, etc.

Los Recursos de Enseñanza disponibles, principalmente el texto escolar seleccionado, materiales didácticos, elementos tecnológicos, el propio entorno, etc. constituyen otro aspecto muy importante a la hora de planificar.

Las características del grupo o del curso, el contexto sociocultural de la comunidad en que está inserto, las diferencias individuales de los alumnos, los modos de aprender los mismos contenidos, el nivel cognitivo, las experiencias personales, las capacidades, conocimientos, sentimientos y actitudes, etc. juegan también un rol muy importante al momento de planificar.

Para abordar esta temática desde la realidad del aula, que sin duda es una constante inquietud y preocupación en los docentes, se realizaron encuestas y entrevistas no estructuradas en el marco del trabajo de la tesis de licenciatura "Seleccionar, Organizar y Secuenciar los Contenidos para Enseñar Geografía".

Luego de haber analizado estos ítems en la encuesta y considerando también las entrevistas no estructuradas mantenidas con varios de los docentes encuestados, se realiza este pequeño aporte en el que, a modo de sugerencias, se presentan pautas para elaborar secuencias de contenidos.

\section{Pautas (sugerencias - formas) para elaborar secuencias de contenidos \\ 1. Partir de los conocimientos previos de los alumnos.}

Se ha comentado en muchas ocasiones que nuestros alumnos no son "pizarrones" en blanco que los docentes, partiendo de la nada, nos dedicamos a rellenar con esmero. Los alumnos construyen 0 , mejor, reconstruyen los contenidos que se les propone aprender, otorgándoles un significado propio sobre la base de las adquisiciones que ya poseen, es decir, partiendo de su propia estructura de conocimientos que está relacionada con el contenido concreto objeto de aprendizaje. D. Ausubel lo resalta con esta sugerente frase: "Si tuviera que reducir toda la psicología evolutiva a un solo principio, enunciaría éste: El factor particular que más influye en el aprendizaje es lo que el alumno ya sabe. Averígüese esto y enséñesele en consecuencia."

Cuando nos referimos a los conocimientos previos de los alumnos, es preciso considerar que, además de la información que ya posean sobre un tema de estudio determinado, que sin duda para la elaboración de secuencias por parte de los docentes es la principal referencia inicial, también sería preciso tener en cuenta sus destrezas, habilidades, estrategias para el estudio (contenidos con un marcado carácter

"procedimental") y, junto con ello, lo que se ha definido como la disposición para abordar el contenido propuesto (motivación, autoconcepto, interés, creencias, etc.).

Si aceptamos una concepción activa del aprendizaje, en la que es el propio alumno quien, partiendo de su propia experiencia reconstruye los significados culturales, debemos convenir a continuación que esta reconstrucción será tanto más fácil para él cuanto mejor organizado esté el contenido que se le propone aprender. Los docentes, en tal caso, deberían diseñar procesos de aprendizaje donde las distancias entre lo que el alumno sabe y lo que se le propone aprender sean adecuadas, sin saltos bruscos entre los contenidos y sin lagunas que dificulten una progresión adecuada en el tratamiento de los mismos.

2. Analizar el contenido considerando el desarrollo de las capacidades que precisan los alumnos para realizar los aprendizajes.

Publicado en formato digital: Prof./ Lic. Lucía Iris Meretz. LA DIFICIL TAREA DE SELECCIONAR, ORGANIZAR Y SECUENCIAR LOS CONTENIDOS AL MOMENTO DE PLANIFICAR. Los Temas Escolares de la Geografía. Revista Geográfica Digital. IGUNNE. Facultad de Humanidades. UNNE. Año 10. No 20. Julio Diciembre 2013. ISSN 1668-5180 Resistencia, Chaco. En: http://hum.unne.edu.ar/revistas/geoweb/default.htm 
En este criterio destaca el análisis del contenido desde el punto de vista del alumno, dándoles, una mayor relevancia a las condiciones necesarias para que el conocimiento sea reconstruido mediante los aprendizajes escolares.

Es aceptado por todos que los alumnos requieren una determinada "madurez" para asimilar determinados contenidos. En algunos casos, nos estamos refiriendo simplemente a la necesidad de que hayan adquirido previamente los conocimientos básicos precisos. Pero generalmente lo que queremos indicar es que los alumnos precisan un determinado desarrollo intelectual relacionado con su desarrollo evolutivo, de manera que les permita haber alcanzado una capacidad intelectual adecuada para asimilar los contenidos propuestos.

Sin embargo, cuando tratamos de concretar esta idea inicial aparecen distintas propuestas. Por un lado, hay autores que otorgan más importancia al desarrollo de capacidades observables mediante tareas, de manera que proponen la elaboración de jerarquías de acciones que comienzan por las más sencillas hasta remontarse a las más complejas. Otros autores otorgan mayor importancia a la reestructuración de esquemas de conocimiento, que están organizados partiendo desde los contenidos más generales para ir derivando progresivamente hacia los más particulares (Ausubel).

En cualquier caso, tanto unos como otros admiten que el alumno pasa por una serie de estadios evolutivos que influyen en la construcción de los aprendizajes, y por tanto deberíamos tenerlos en cuenta a la hora de diseñar la enseñanza de un conjunto de contenidos.

Deberíamos complementar, por tanto, el análisis lógico del contenido que vamos a secuenciar con el análisis psicológico del mismo, es decir, adecuar el tratamiento del contenido a las posibilidades de aprendizaje que permite en cada momento el desarrollo de los alumnos a los que se dirige. Para ello, diversos estudios de psicología nos aportan referencias concretas que permiten saber cuándo los alumnos pueden asimilar con más garantías y eficacia conceptos como: volumen, cantidad, energía, concepción del espacio, etc.

\section{Elegir un contenido organizador.}

En el proceso de elaboración de secuencias, no podemos utilizar como referente organizador de la misma los distintos tipos de contenidos al mismo tiempo (conceptos, procedimientos y actitudes), ya que será muy difícil, cuando no imposible, encontrar criterios que permitan determinar y fundamentar la conveniencia de abordar unos contenidos antes que otros, indicando por ejemplo si se ha de tratar antes un concepto que una actitud, o un procedimiento. Es preciso, por tanto, que, para organizar los contenidos de un modo coherente se actúe inicialmente sobre un solo tipo de contenido, conceptual, procedimental o actitudinal, de manera que el contenido elegido sirva de organizador y vertebrador de toda la secuencia, y posteriormente en torno al mismo se irán distribuyendo el resto de contenidos de los otros tipos.

Recordamos que Ausubel propone los conceptos como contenido organizador, pues piensa que nuestra estructura de conocimiento está formada por conceptos y las numerosas relaciones que se establecen entre ellos. De manera que tomaría como punto de partida los conceptos más generales e inclusores, y a partir de ellos irían derivando el resto de conceptos más particulares relacionados con aquellos mediante jerarquías conceptuales.

\section{Tener en cuenta todos los tipos de contenidos}

Si bien es cierto que cada cuerpo de contenidos se presta más, como acabamos de ver, a la elección de un contenido organizador, para que los alumnos respondan globalmente ante los aprendizajes propuestos, necesitan abordar los distintos tipos de contenidos de un modo equilibrado. Los contenidos que acompañan al organizador en el transcurso de la secuencia son los contenidos de "soporte", de tal manera que si elegimos por ejemplo los conceptos, sería necesario posteriormente acompañarlos de los procedimientos que el alumno va a necesitar para abordarlos, y del desarrollo de las actitudes que promueve su tratamiento.

$\mathrm{Si}$ se estudian conjuntamente y de modo relacionado los contenidos conceptuales, procedimentales y actitudinales, se facilitará su asimilación por parte de los alumnos, que les confieren así un mayor sentido al contemplarlos desde la globalidad de sus creencias, actitudes, conocimientos, etc. 
Precisamente, una de las claves de la aplicación de enfoques globales en el aula y dentro de un currículo integrado está precisamente en que promueven la planificación de los distintos tipos de contenidos de un modo interrelacionado, superando así otros modelos en los que generalmente prevalecía el contenido conceptual, hasta el punto de anular en muchos casos los contenidos procedimentales y actitudinales, que hoy consideramos de capital importancia en los procesos de aprendizaje de los alumnos. Por tanto, no deberíamos realizar una separación artificial de los mismos si no es a efectos meramente de estudio y planificación previa al trabajo de aula, ya que, como hemos indicado, en el proceso de enseñanza/aprendizaje nuestros alumnos trabajarán los distintos tipos de contenidos de una manera complementaria, global.

En relación a esto podemos decir que los alumnos responden a los aprendizajes propuestos desde tres niveles, que tienen un carácter complementario, y son:

* Un primer nivel corresponde a la adquisición de conocimientos principalmente de tipo conceptual, ampliando las redes y esquemas que ya posee el alumno mediante la incorporación de nuevos contenidos que van a facilitar una mejor interpretación de la realidad.

- Pero, para que el alumno realice adecuadamente el tratamiento de la información que recibe, es preciso que desarrolle destrezas y procedimientos que permitan su recopilación, clasificación, interpretación, expresión, etc., adquiriendo y mejorando las estrategias de aprendizaje que le ayuden a aprender por sí mismo.

* Sin embargo, el nivel más profundo desde el que responden los alumnos ante un aprendizaje es aquél relacionado con sus vivencias internas, con sus concepciones íntimas y personales sobre los acontecimientos y fenómenos que le rodean. Estos contenidos, que generalmente han formado parte del llamado "currículo oculto", se agrupan en el currículo con el nombre de actitudes, y ocupan un lugar cada vez más destacado en la planificación de la enseñanza, ya que de poco sirve que los alumnos adquieran conocimientos conceptuales y procedimentales, si no tienen una actitud positiva ante lo aprendido.

Estas reflexiones proponen con claridad que una secuencia bien elaborada necesita incluir la planificación de todos los tipos de contenidos, con el fin de facilitar el acercamiento al conocimiento del modo más completo y global posible.

\section{Ejes de contenidos}

Todas las secuencias incluyen una gran cantidad de contenidos, de modo que uno de los problemas que presenta su utilización es la dificultad para orientarse dentro de ella. Por tanto, una vez elegido el contenido organizador, conviene definir seguidamente unos ejes en torno a los cuales se van a distribuir todos los contenidos de la secuencia, y que representan el armazón organizativo de la misma.

A través de estas grandes vías de comunicación de la secuencia, se podrá realizar una lectura rápida de la misma, y proporcionan la orientación necesaria para encontrar un contenido determinado con facilidad, favoreciendo así el desarrollo de las actividades programadas en el aula, lo que influirá decisivamente en que la misma secuencia tenga una mayor utilidad y se frecuente más su uso por parte del docente.

\section{Organizar los contenidos siguiendo una progresión de dificultad en su tratamiento.}

Los criterios para definir la progresión que deben seguir los contenidos dependerá en cada caso del referente que utilicemos. Por ejemplo, si se trata de trabajar contenidos relacionados con las escalas espaciales, puede ser recomendable que progresemos desde lo más cercano a la experiencia del alumno, hacia lo más alejado de la misma. Si abordásemos contenidos conceptuales, sería conveniente proceder desde lo más general hacia lo más particular. Si se tiene en cuenta el factor referido a los conocimientos previos, deberíamos proceder desde lo más conocido por el alumno hacia lo que le resulta más desconocido. Tendiendo al grado de complejidad, podríamos acometer el estudio de los contenidos desde lo más concreto hacia lo más abstracto, y así sucesivamente.

En cualquier caso, siempre se debe procurar diseñar la enseñanza siguiendo una progresión que parta desde lo más sencillo hacia lo más complejo, de manera que una secuencia bien diseñada tiene que reflejar con claridad esta progresión de contenidos para facilitar los aprendizajes de los alumnos, evitando saltos y retrocesos que lo obstaculicen.

Publicado en formato digital: Prof./ Lic. Lucía Iris Meretz. LA DIFICIL TAREA DE SELECCIONAR, ORGANIZAR Y SECUENCIAR LOS CONTENIDOS AL MOMENTO DE PLANIFICAR. Los Temas Escolares de la Geografía. Revista Geográfica Digital. IGUNNE. Facultad de Humanidades. UNNE. Año 10. № 20. Julio Diciembre 2013. ISSN 1668-5180 Resistencia, Chaco. En: http://hum.unne.edu.ar/revistas/geoweb/default.htm 


\section{Realizar un tratamiento cíclico de los contenidos.}

La organización de los contenidos sugerida en el criterio anterior no debe entenderse como una progresión lineal, sino que el tratamiento de los contenidos debe ser cíclico, es decir, que en muchos casos éstos no se trabajarán en un solo ciclo y por una única vez, sino que se irán abordando en sucesivos niveles de complejidad a lo largo de los distintos ciclos de la etapa, en estrecha relación con el nivel de desarrollo y conocimientos de los alumnos.

Es conveniente indicar también que en una secuencia no sólo debemos tener en cuenta este criterio de organización cíclica de los contenidos para referirnos al aumento cuantitativo de los mismos, ampliando sucesivamente los atributos en los conceptos estudiados al progresar en los ciclos de la etapa, o bien en relación con los procedimientos ir aumentando el número de acciones que se precisa realizar en su aplicación, etc., sino que también es necesario contemplar la complejidad de las relaciones que establecen a su vez con otros contenidos, o secuencias, es decir, deberíamos tener en cuenta su mayor poder explicativo de la realidad que se orienta hacia una comprensión más global del conocimiento.

\section{Es conveniente que la secuencia adopte una estructura que sea fácil de modificar por el equipo docente}

Finalmente, lo importante de una secuencia de contenidos de una etapa educativa no es la realización de un ejercicio teórico por parte del equipo docente, sino que se transforme en un instrumento de planificación que facilite la tarea de diseñar el proceso de enseñanza/aprendizaje en las aulas. Para ello, una secuencia debe ser fácil de aplicar y, a la vez, debería ser fácil de modificar, incorporando aquellas rectificaciones extraídas de la práctica diaria en el aula, que es el lugar donde las previsiones de la secuencia se someten a constante evaluación.

Por tanto, una secuencia en la que solamente se incluye una distribución de contenidos por ciclos, presentados en bloques sin conexión entre ellos, sin un contenido organizador que aporte coherencia a esa distribución y sin unos ejes que permitan una vertebración adecuada de la misma, lo que nos proporciona son unos conjuntos de contenidos con un carácter muy estático, donde la única posibilidad de modificación que nos permiten consiste en pasar contenidos de un ciclo a otro sin determinar una adecuada ubicación dentro de los mismos y sin las relaciones necesarias que contribuyan a explicarlos.

Ahora bien, cuando una secuencia nos ofrece un orden en los contenidos a lo largo de la etapa y, sobre todo, presenta las relaciones que se establecen entre ellos, en este caso el docente puede introducir modificaciones parciales surgidas puntualmente como consecuencia de la evaluación diaria de su trabajo en clase, puede dar lugar a través del tiempo a una reconstrucción progresiva de toda la secuencia, convirtiéndola en un instrumento más personal de planificación, y por tanto más útil.

\section{CONCLUSIÓN}

La ciencia geográfica, como todas las ciencias, ha variado en sus enfoques según los contextos en los que se desarrolló. Esos contextos abarcan hechos sociales, políticos, económicos, culturales y científicos, que influyeron en la definición de objetivos, sus características conceptuales y metodológicas, tanto de estudio como de enseñanza (Vilá Valentí, 1983).

En un contexto de acelerados cambios sociales, económicos, políticos, culturales y ambientales se vislumbra, en forma apremiante, la idea de que la enseñanza de la geografía no puede desentenderse de los múltiples aportes provenientes de los distintos ámbitos de la producción científica y cultural. En este sentido, el análisis y la discusión de nuevos marcos interpretativos y de herramientas conceptuales que permitan comprender y explicar esas transformaciones se constituyen en una instancia clave para habilitar la revisión de los criterios con los que seleccionar contenidos escolares relevantes y útiles. .

Se bosqueja entonces la premisa acerca de que la construcción del conocimiento escolar requerirá, en cada contexto particular de enseñanza, de una serie de mediaciones pedagógicas que transformen los saberes provenientes del campo disciplinar y cultural en contenidos susceptibles de 
ser enseñados, aprendidos y evaluados. Mediaciones que se concretan en recortes temáticos, estudios de caso o de profundizaciones singulares referidas a los temas de interés geográfico.

En este proceso de transposición didáctica, la problematización de los contenidos comienza a perfilarse como uno de los caminos más potentes para transitar esas mediaciones, atendiendo a los criterios de validez, relevancia y significatividad de los temas que se aborden en las clases.

La renovación didáctica asume también la idea de que la selección de contenidos no es independiente de su tratamiento metodológico, porque ofrecer a los alumnos y alumnas contenidosproblema con suficiente potencialidad pedagógica para lograr aprendizajes significativos conlleva una rigurosa elección de aquellas temáticas geográficas sobre las que la sociedad formula preguntas y dilemas de conocimiento y sobre las que el sistema educativo ensaya estrategias didácticas para su tratamiento en clase.

Como los conceptos en ciencias sociales son susceptibles de distintos abordajes y desarrollos, no alcanzaría con decir que en las clases de geografía se estudiarán, por ejemplo, las regiones para dar a entender qué es lo que realmente se va a enseñar, porque no existe un significado único del concepto de región y para definirla es necesario tener en cuenta distintos criterios de regionalización y hacerlos explícitos.

Si una de las tendencias de las ciencias sociales actuales es reconocer y respetar la coexistencia de diferentes enfoques, la enseñanza de la geografía ha de dar cuenta de esa tendencia presentándolos como discursos a ser examinados. Esta posición plantea exigencias epistemológicas, porque obliga a considerar la variedad de perspectivas que estudian los procesos socio-territoriales y a observar que cada opción teórica acarrea determinadas alternativas de acción desde el punto de vista pedagógico-didáctico.

Una condición para que la selección y el tratamiento de los temas se ajusten al mosaico geográfico en permanente transformación es apelar a un modo de conocer atento a esos movimientos desde los principios de complejidad, multiperspectividad, diversidad, cambio, incertidumbre, controversia y pluralismo.

Explicar cuestiones relativas a los recortes particulares que la didáctica exige realizar también nos lleva a considerar la diferencia entre contenidos de los campos disciplinarios e inventarios organizados para la enseñanza (Litwin, 1996). 
Revista Geográfica Digital. IGUNNE. Facultad de Humanidades. UNNE. Año 10. № 20. Julio - Diciembre 2013. ISSN 1668-5180 Resistencia, Chaco

\section{Referencias}

1.- BLANCO, J. y OTROS. 1997. Notas sobre la enseñanza de una Geografía renovada. $4^{a}$ edic.Aique Grupo Editor. Buenos Aires. pág. 25

2.- CORDERO, Silvia y SVARZMAN, José. 2007. Hacer Geografía en la Escuela. Reflexiones y aportes para el trabajo en el aula. Ediciones Novedades Educativas. $1^{\text {a }}$ Edic. Buenos Aires.

3.- CORDERO, Silvia y SVARZMAN, José. 2007 .Hacer Geografía en la Escuela. Reflexiones y aportes para el trabajo en el aula. Ediciones Novedades Educativas. $1^{\text {a }}$ Edic. Buenos Aires.

4.-GALLOPIN, G. "Tecnología y sistemas ecológicos". En Boletín de Medio Ambiente y Urbanización. Nº 12

5.- Ibidem

6.- GALLOPIN, G. "Tecnología y sistemas ecológicos". En Boletín de Medio Ambiente y Urbanización. № 12.

7.- BLANCO, J. y OTROS. 1997. Notas sobre la enseñanza de una Geografía renovada. $4^{a}$ edic. Aique Grupo Editor. Buenos Aires. pág. 155

8.- BAILEY, Patrick. 1981. Didáctica de la Geografía. Trad. Eduardo Sierra Valenti. Edit. Cincel Kapelusz. Madrid. España. Pág. 33

9.- CORDERO, Silvia, SVARZMAN, José. 2007Hacer Geografía en la Escuela. Reflexiones y aportes para el trabajo en el aula. Ediciones Novedades Educativas. 1ª Edic. Buenos Aires.

\section{BIBLIOGRAFIA}

1. ANDER-EGG, Ezequiel. La Planificación Educativa. Conceptos, métodos, estrategias y técnicas para educadores. $10^{\mathrm{a}}$ reimpresión. Edit. Magisterio del Río de la Plata. Buenos Aires. 2008.

2. BAILEY, Patrick. Didáctica de la Geografía. Trad. Eduardo Sierra Valenti. Edit. Cincel Kapelusz. Madrid. España. 1981.

3. BLANCO, J. y OTROS. Notas sobre la enseñanza de una Geografía renovada. $4^{a}$ edic. Aique Grupo Editor. Buenos Aires. 1997.

4. CALAF MASACHS, Roser y OTROS. Aprender a enseñar Geografía. Edit. Oikos-tau. Barcelona. España. 1997.

5. COLL, César y OTROS. Los contenidos en la reforma. Enseñanza y aprendizaje de conceptos, procedimientos y actitudes. Edit. Santillana. Buenos Aires. 1994.

6. CORDERO, Silvia, SVARZMAN, José. Hacer Geografía en la Escuela. Reflexiones y aportes para el trabajo en el aula. Ediciones Novedades Educativas. 1ª Edic. Buenos Aires. 2007.

7. DAVINI, María Cristina. Métodos de enseñanza. Edit. Santillana. Buenos aires. 2008.

8. DURAN, Diana y OTROS. Los cambios mundiales y la enseñanza de la geografía. $1^{\mathrm{a}}$ edic. Edit. Troquel. Buenos Aires. 1993.

9. DURAN, Diana. Geografía y Transformación Curricular. Ministerio de Cultura y Educación de la Nación. Lugar Editorial. Buenos Aires. 1996.

10. ESTEBANEZ, José. Tendencias y Problemática actual de la Geografía. 10ª reimpresión. Edit. Cincel. Madrid. 1990.

11. FERNANDEZ CASO, M. victoria (Coord.). Geografía y territorios en transformación. Nuevos temas para pensar la enseñanza. Ediciones Novedades Educativas. Buenos Aires. Abril 2007.

12. FERNANDEZ CASO, M. Victoria, GUREVICH, Raquel (coord.). Geografía. Nuevos temas, nuevas preguntas. Un temario para su enseñanza. Editorial Biblos. Buenos Aires. 2007.

13. FERRERO, A.; MORINA, J.O.; VILLA, A., "La Geografía en la escuela media: una propuesta de enfoque". Actas de las Séptimas Jornadas Cuyanas de Geografía. Universidad Nacional de Cuyo. Mendoza. 1992.

14. FERRERO, Adrián Nicolás. La Construcción del conocimiento geográfico. Estrategias didácticas para el $3^{\circ}$ ciclo y Polimodal. Homo Sapiens Ediciones. Rosario. Argentina. 1998.

15. GRAVES, Norman J. La enseñanza de la geografía. Trad. Genís Sánchez. $1^{a}$ ed. En castellano. Edit. Visor. España 1985.

16. GUREVICH, Raquel y OTROS. Notas sobre la enseñanza de una geografía renovada. $4^{\mathrm{a}}$ edic. Edit. Aique. Buenos Aires. 1997.

17. HARF, Ruth. 1996. "Poniendo la Planificación sobre el Tapete". Documento de trabajo. MCBA.

18. LITWIN, Edith. (1997). Las configuraciones didácticas. Una nueva agenda para la enseñanza superior. $1^{\mathrm{a}}$ edic. Edit. Paidós Educador. Buenos Aires.

Publicado en formato digital: Prof./ Lic. Lucía Iris Meretz. LA DIFICIL TAREA DE SELECCIONAR, ORGANIZAR Y SECUENCIAR LOS CONTENIDOS AL MOMENTO DE PLANIFICAR. Los Temas Escolares de la Geografía. Revista Geográfica Digital. IGUNNE. Facultad de Humanidades. UNNE. Año 10. No 20. Julio Diciembre 2013. ISSN 1668-5180 Resistencia, Chaco. En: http://hum.unne.edu.ar/revistas/geoweb/default.htm 
19. MINISTERIO DE CULTURA Y EDUCACIÓN DE LA NACIÓN. Consejo Federal de Cultura y Educación. Contenidos Básicos Comunes para la Educación General Básica. $2^{\mathrm{a}}$ edic. Buenos Aires. 1995.

20. MINISTERIO DE CULTURA Y EDUCACIÓN DE LA NACIÓN. Consejo Federal de Cultura y Educación. Contenidos Básicos para la Educación Polimodal. Buenos Aires. 1997.

21. SANCHEZ INIESTA, Tomás. Organizar los contenidos para ayudar a aprender. Un modelo de secuencia de los Contenidos Básicos comunes. $2^{\mathrm{a}}$ edic. Edit. Magisterio del Río de la Plata. Buenos Aires. 1999.

22. SPIEGEL, Alejandro. Planificando clase interesantes. Itinerarios para combinar recursos didácticos. Ediciones Novedades Educativas. Buenos Aires. 2006. 